\title{
Breast cancer identification via modeling of peripherally circulating miRNAs
}

\author{
Xiaomeng Cui ${ }^{1,2}$, Zhangming Li ${ }^{3}$, Yilei Zhao ${ }^{4}$, Anqi Song ${ }^{5}$, Yunbo Shi ${ }^{\text {Corresp., }}{ }^{1,2}$, Xin Hai ${ }^{4}$, Wenliang Zhu ${ }^{6}$ \\ ${ }^{1}$ The higher educational key laboratory for Measuring \& Control Technology and Instrumentations of Heilongjiang Province, Harbin, China \\ 2 School of Measurement-Control Tech \& Communications Engineering, Harbin University of Science and Technology, Harbin, China \\ 3 Department of Pharmacy, Guangdong Hospital of Integrated Chinese and Western Medicine, Foshan, China \\ 4 Department of Pharmacy, First Affiliated Hospital of Harbin Medical University, Harbin, China \\ 5 Department of Student Affairs, Second Affiliated Hospital of Harbin Medical University, Harbin, China \\ 6 Department of Pharmacy, Second Affiliated Hospital of Harbin Medical University, Harbin, China \\ Corresponding Author: Yunbo Shi \\ Email address: shiyunbo@hrbust.edu.cn
}

Prolonged life expectancy in humans has been accompanied by an increase in the prevalence of cancers. Breast cancer (BC) is the leading cause of cancer-related deaths. It accounts for one fourth of all diagnosed cancers and affects one in eight females worldwide. Given the high BC prevalence, there is a practical need for demographic screening of the disease. In the present study, we re-analyzed a large microRNA (miRNA) expression dataset (GSE73002), with the goal of optimizing miRNA biomarker selection using neural network cascade (NNC) modeling. Our results identified numerous candidate miRNA biomarkers that are technically suitable for BC detection. We combined three miRNAs (miR-1246, miR-6756-5p, and miR-8073) into a single panel to generate a NNC model, which successfully detected BC with $97.1 \%$ accuracy in an independent validation cohort comprising 429 BC patients and 895 healthy controls. In contrast, at least seven miRNAs were merged in a multiple linear regression model to obtain equivalent diagnostic performance (96.4\% accuracy in the independent validation set). Our findings suggested that suitable modeling can effectively reduce the number of miRNAs required in a biomarker panel without compromising prediction accuracy, thereby, increasing the technical possibility of early detection of BC. 


\section{Breast cancer identification via modeling of peripherally circulating miRNAs}

2 Xiaomeng Cui ${ }^{1,2}$, Zhangming $\mathrm{Li}^{3}$, Yilei Zhao ${ }^{4}$, Anqi Song ${ }^{5}$, Yunbo Shi ${ }^{1,2, *}$, Xin Hai ${ }^{4, *}$, Wenliang Zhu ${ }^{6}$

$3 \quad{ }^{1}$ The higher educational key laboratory for Measuring \& Control Technology and Instrumentations of

4 Heilongjiang Province, Harbin, 150080, China

$5 \quad{ }^{2}$ School of Measurement-Control Tech \& Communications Engineering, Harbin University of Science and

6 Technology, Harbin, 150080, China

$7{ }^{3}$ Department of Pharmacy, Guangdong Hospital of Integrated Chinese and Western Medicine, Foshan, 8528200, China.

$9 \quad{ }^{4}$ Department of Pharmacy, First Affiliated Hospital of Harbin Medical University, Harbin, 150001, China.

${ }^{5}$ Department of Student Affairs, Second Affiliated Hospital of Harbin Medical University, Harbin 150086,

11 China.

${ }^{6}$ Department of Pharmacy, Second Affiliated Hospital of Harbin Medical University, Harbin 150086,

China.

*To whom correspondence should be addressed. Yunbo Shi: Address: 52 Xuefu Road, Harbin 150080, 


\section{ABSTRACT}

19 Prolonged life expectancy in humans has been accompanied by an increase in the prevalence of cancers.

Breast cancer (BC) is the leading cause of cancer-related deaths. It accounts for one fourth of all diagnosed

cancers and affects one in eight females worldwide. Given the high BC prevalence, there is a practical need

for demographic screening of the disease. In the present study, we re-analyzed a large microRNA (miRNA) expression dataset (GSE73002), with the goal of optimizing miRNA biomarker selection using neural network cascade (NNC) modeling. Our results identified numerous candidate miRNA biomarkers that are technically suitable for BC detection. We combined three miRNAs (miR-1246, miR-6756-5p, and miR8073 ) into a single panel to generate a NNC model, which successfully detected BC with $97.1 \%$ accuracy in an independent validation cohort comprising $429 \mathrm{BC}$ patients and 895 healthy controls. In contrast, at least seven miRNAs were merged in a multiple linear regression model to obtain equivalent diagnostic performance (96.4\% accuracy in the independent validation set). Our findings suggested that suitable modeling can effectively reduce the number of miRNAs required in a biomarker panel without compromising prediction accuracy, thereby, increasing the technical possibility of early detection of BC. 


\section{INTRODUCTION}

34 Breast cancer $(\mathrm{BC})$ is one of the most common cancers that accounts for one in four diagnosed cancers and

affects one in eight females worldwide (Torre et al., 2015). Approximately 1.5 million new BC cases are

reported per year (Siegel et al., 2015), which is close to the existing 1.7 million BC cases reported in 2012.

Conservative estimates suggested higher morbidity rates associated with $\mathrm{BC}$ though only prolonged life

expectancy of females was considered. Therefore, early demographic screening is necessary to manage the

unprecedented increase in the malignant disease (Myers et al., 2015). However, currently employed BC

screening methods have relatively low sensitivity and insufficient identification power, leading to a high

false positive rate of $20.5 \%$ in women aged $40-49$ years (van den Ende et al., 2017). Therefore, there is a

need for the development of novel biomarkers for early detection of BC.

MicroRNAs (miRNAs) are a class of single-stranded small non-coding RNA molecules of $\sim 22$

nucleotides. miRNAs act as post-transcriptional gene expression regulators via complementary binding to

the 3'-untranslated regions of mRNAs (Bartel, 2009). Recent studies have shown important involvement of

miRNAs in the pathological process of $\mathrm{BC}$ via regulating proliferation and energy synthesis of $\mathrm{BC}$ cells $(\mathrm{Li}$

et al., 2017; Chen et al., 2018; Xiao et al., 2018). The miRBase database currently includes data on more

than 2800 mature human miRNAs (Kozomara and Griffiths-Jones, 2014). Of these, some miRNAs, such

as miR-21 and miR-155, have demonstrated potential value for the early diagnosis of BC (Hamam et al.,

2017). Meanwhile, the development of new detection techniques made accurate detection of low-abundance

circulating miRNAs no longer an obstacle (Majd et al, 2018).

Despite significant progress in research on the use of circulating miRNAs as diagnostic BC biomarkers, 
54

55

56

57

\section{MATERIALS AND METHODS}

reproducibility (Nassar et al., 2017). Thus, there is a need for a systematic review of candidate biomarkers reported in previous clinical studies. BC is considered a collection of mammary gland-related heterogeneous diseases (Bertos and Park, 2011). In addition, the high BC prevalence requires large sample sizes so that multiple types of $\mathrm{BC}$ can be investigated in a single circulating miRNA biomarker study. So far, only one study has met this requirement. In a study comprising approximately 4000 patients and healthy subjects, Shimomura and his colleagues performed a microarray-based circulating miRNA biomarker assay for early detection of BC in the Japanese population (Shimomura et al., 2016). The authors validated the effectiveness of a biomarker panel comprising five miRNAs (miR-1246, miR-1307-3p, miR-4634, miR6861-5p, and miR-6875-5p) for BC diagnosis with $89.7 \%$ accuracy. Surprisingly, the aforementioned five miRNAs were not reported by other studies with small sample sizes (Nassar et al., 2017). Therefore, larger sample sizes can facilitate the discovery of miRNA biomarkers, while smaller sample sizes can introduce more sampling error and inconsistencies in miRNA biomarkers among different studies.

Although the authors provided a valuable data resource for expression levels of circulating miRNAs in BC (GSE73002), no optimization was performed for the miRNA biomarker panel, which could potentially increase diagnostic accuracy. The neural network cascade (NNC) modeling has been demonstrated to have high prediction accuracy than the traditional artificial neural network (ANN) modeling (Li et al., 2015; Hou et al., 2016; Qu et al., 2017). In this study, NNC models were generated to re-analyze the dataset comprising circulating miRNAs in $\mathrm{BC}$ and to optimize the miRNA biomarker panel for early detection of BC. Our approach was effective in identifying suitable diagnostic biomarkers for demographic screening for BC. 


\section{miRNA expression data}

Expression data of circulating miRNAs in BC patients and healthy controls (GSE73002) were retrieved from the Gene Expression Omnibus (GEO) repository (Shimomura et al., 2016). The present study included data from a total of 3974 participants, including 1288 BC patients and 2686 healthy controls. For each participant, the normalized microarray expression values of 2540 miRNAs were downloaded from GEO, and the corresponding disease statuses (healthy: 0 or BC: 1) were obtained for further modeling.

\section{Biomarker evaluation and selection}

Data from the 3974 participants were randomly divided into two sets, namely, a training set $(\mathrm{n}=2650)$ and a validation set $(n=1324)$. Later, each of the 2540 miRNAs in the training set was independently evaluated as a potential miRNA biomarker for BC. We performed receiver operating characteristic (ROC) curve analysis of the miRNA expression values using MedCalc version 15.8 (MedCalc, Mariakerke, Belgium).

We calculated the area under the curve (AUC) to evaluate whether the generated models can reliably distinguish between $\mathrm{BC}$ and healthy controls (AUC $>0.95$ and $p<0.0001$ ). Since all expression values corresponding to miRNA models with AUC $\geq 0.95$ did not satisfy the D'Agostino-Pearson omnibus normality test or follow a Gaussian distribution, we conducted Spearman's correlation tests to determine the collinearities among the miRNAs using GraphPad Prism version 6.0 (GraphPad Software, Inc., La Jolla,

CA, USA). Collinearity was considered significant at the threshold of $|\rho|>0.5$. If the expression values of two miRNAs are collinear $(|\rho|>0.5)$, only the miRNA with a larger AUC was retained for further modeling. A collinearity network of the miRNAs with AUC $\geq 0.95$ was generated using the network visualization software Cytoscape v3.6.0 (Institute of Systems Biology, Seattle, WA, USA) (Shannon et al., 2003).

\section{NNC and multiple linear regression (MLR) models}


96

97

The expression values of the miRNAs with $A U C \geq 0.95$ were normalized to a value between 0 and 1 before further model building, as previously described (Zhu and Kan, 2014). The Intelligent Problem Solver (IPS) tool in the Statistica Neural Networks (SNN, Release 4.0E; Statsoft, Tulsa, OK, USA) software was used to build a radial basis function (RBF)-ANN model with 1-11-1 network architecture to investigate the associations of individual miRNAs with the disease status. Afterwards, an NNC model was built following a step-by-step procedure as previously described (Li et al., 2015). Briefly, the ANN that contributed to the maximum increase in AUC was retained for further extension of the ANN cascade. Such a modeling operation would be terminated artificially until there was no further increase in AUC or all of the miRNAs were incorporated in the NNC model. For comparison, a MLR model was also built based on the miRNAs with AUC $\geq 0.95$ using the SPSS statistical software version 19.0 (IBM Corp., New York city, NY, USA).

\section{Model validation}

A hold-out cross-validation method was used for internal validation of each of the RBF-ANNs in the NNC model. Briefly, IPS divided the modeling set into three subsets (training subset, verification subset, and testing subset) at a 2:1:1 ratio. Data on participants included in the testing subset were not used for model building but were used for model validation. The correlation coefficients given by IPS were compared to those from the training subset $\left(\mathrm{R}_{\mathrm{Tr}}\right)$; the testing subset $\left(\mathrm{R}_{\mathrm{Te}}\right)$ measured the linear relationship between the model output values and the normalized miRNA expression values. Similar $\mathrm{R}_{\mathrm{Te}}$ and $\mathrm{R}_{\mathrm{Tr}}$ values indicated good generalizability of the corresponding RBF-ANN. Furthermore, a tenfold cross-validation method was used to validate the NNC model. The entire training set $(\mathrm{n}=2650)$ was randomly divided into ten mutually exclusive groups of nearly equal size. Nine of the groups were selected for model training, while the remaining group was used for model validation. The above procedure was repeated ten times, as previously 
117

118

described (Li et al., 2015). Furthermore, an independent validation set $(\mathrm{n}=1324)$ was used for external validation of the NNC and MLR models. Three parameters, namely, sensitivity, specificity, and accuracy rate, were used for model evaluation and validation. Sensitivity was calculated as the percentage of the number of true positives divided by the sum of true positives and false negatives. Specificity was calculated as the percentage of number of true negatives divided by the sum of true negatives and false positives. Accuracy rate (ACC) was calculated as the number of successfully identified BC patients and healthy controls divided by the sum of all the participants.

\section{Data statistics}

Spearman's correlation test was conducted using Graphpad Prism v6.0. ROC curve analysis was performed using MedCalc v13.0. Statistically significant differences were considered at $p<0.0001$.

\section{RESULTS}

\section{High AUCs revealed the redundancy of technically suitable miRNA biomarkers for BC}

In the present study, data of 3974 participants were obtained from the GSE73002 dataset. All participants were randomly assigned into two sets, namely, the training set and validation set, at a 2:1 ratio. The training set consisted of $859 \mathrm{BC}$ patients and 1791 healthy controls. The validation set comprised $429 \mathrm{BC}$ patients and 895 healthy controls. We then investigated the technical feasibility of each of the 2540 miRNAs for $\mathrm{BC}$ identification in each of the training sets. Figure 1A shows the frequency distribution of the AUC values calculated from ROC curve analyses. Approximately $74 \%$ of all miRNAs showed high AUC values (AUC $>0.9$ ), which indicated the strong reliability of the generated models for BC detection. A total of 82 miRNAs with high AUC values are highlighted (AUC >0.95). Furthermore, we observed consistent collinearity 
among the 82 miRNAs (Figure 1B), which implied very high redundancy of candidate miRNA biomarkers

used for BC detection. Finally, we identified eight non-collinear miRNAs that satisfied AUC $>0.95$. These miRNAs are listed in Table 1. Compared with the seven other miRNAs, miR-8073 showed the highest AUC value (AUC=0.991) and the highest accuracy for identifying $\mathrm{BC}$ in the training set $(\mathrm{ACC}=97.0 \%)$.

NNC model integrating three miRNAs for BC detection

An NNC model was built to generate a miRNA biomarker panel for BC diagnosis using the eight miRNAs

listed in Table 1. Finally, three miRNAs, namely, miR-1246, miR-6756-5p, and miR-8073, were used to effectively extend the cascade (Figure 2A). The NCC consisted of three 1-11-1 RBF-ANN units and two 2-11-1 RBF-ANN units. Each of the five RBF-ANNs showed similar $\mathrm{R}_{\mathrm{Te}}$ and $\mathrm{R}_{\mathrm{Tr}}$ values. Furthermore, an MLC model was built by considering the same eight miRNAs as candidate model inputs. Except for miR6748-5p, seven miRNAs were automatically selected into an MLR model using the SPSS software. Although only three miRNAs were included in the NNC model and four additional miRNAs were used in the MLR model, significant differences were not observed between the NNC and MLR models. Table 2 lists the core evaluation parameters for the two models. Although both models had the same AUC, the NNC model showed better performance for BC identification. The accuracy rate of the NNC model was $98.5 \%$, while that of the MLR model was $97.4 \%$.

\section{NNC successfully identified $\mathrm{BC}$ in the validation set}

To validate the effectiveness of the NNC model for BC identification, we performed a tenfold crossvalidation. The NNC model had an AUC of 0.995, which demonstrated its effectiveness for BC detection (Figure 3A). An independent validation set consisting of data from 1324 participants was used to further validate the NNC model. The NNC model was found to have an AUC similar with those of the single 
159 160

miRNA (miR-8073) model and the MLR model (Figure 3B). However, the NNC model actually showed the highest accuracy for $\mathrm{BC}$ identification ( $\mathrm{ACC}=97.1 \%$; Figure $3 \mathrm{C})$. The sensitivity and specificity of the NNC model were $96.7 \%$ and $97.2 \%$, respectively.

\section{DISCUSSION}

The GSE73002 breast cancer (BC) dataset, comprising data from a Japanese population, is the largest miRNA dataset published in GEO. China has also witnessed an increase in the number of BC cases in recent years (Jiang et al., 2018). BC has become the most prevalent malignant disease in Chinese females, with nearly 270,000 new BC cases reported in 2015. Consequently, BC has become a serious and widespread social issue that cannot be solved by treatment alone. Early detection of $\mathrm{BC}$ in the population represents an optimal strategy for improving the survival rates of BC patients (Sun et al., 2017).

Considerable evidence has demonstrated the technical reliability of miRNAs as early diagnostic markers for BC because of their relatively simple molecular structure and stability (Bahrami et al., 2018). However, multiple studies have not agreed upon a consensus set of miRNAs that are useful as biomarkers, which could be attributed to inadequate sample sizes in the majority of studies (Nassar et al., 2017). The above findings indicated that cohorts with small samples sizes within the range of dozens to hundreds are of little or no value for the identification of potential miRNAs as early diagnostic biomarkers. Therefore, simply counting the number of times a miRNA was validated as a BC biomarker in different studies is not reliable. The heterogeneity of different $\mathrm{BC}$ subtypes is a major consideration for the initial research design (Yeo and Guan, 2017). The sample size is a crucial design parameter for a clinical study. Inadequate samples do not fully represent the whole population (Freiman et al., 1978). However, large sample sizes are difficult to 
180

181

obtain because of certain criteria that limit the number of available samples. For example, research funding may not be sufficient to support a large sample size, and different research groups may have limited staff for implementation of the research protocols. Moreover, research groups are usually relatively isolated from each other and most studies tend to address problems in the regions in which the studies were performed.

Re-analysis of the GSE73002 dataset facilitated the selection and optimization of biomarkers from the human miRNAome, a sample set that is most representative of the population. One of our main findings is that circulating miRNAs serve as highly useful markers for BC detection. A single miRNA can be used as a biomarker without the need for data reprocessing. For example, our models achieved close to $96 \%$ accuracy in the independent validation set using miR-8073 as the biomarker. Our results were consistent with those of previously reported miRNA biomarkers (Nassar et al., 2017).

In addition, our findings showed that appropriate data modeling is necessary for optimization of miRNA biomarkers. The currently developed NNC model showed significantly higher accuracy ranging from $95.8 \%$ to $97.1 \%$ after integration of single miRNA, miR-8073, with the two miRNAs miR-6756-5p and miR-1246 that was validated as biomarkers for several cancers (Hannafon et al., 2016; Machida et al., 2016;

Todeschini et al., 2017). However, six additional miRNAs were needed to achieve the same accuracy rate when using the MLR model. The MLR model is a widely used mathematical model that can be used to construct miRNA biomarker panels for the detection of various human diseases (Ding et al., 2017). The NNC is a tandem mode of multiple small ANNs that generates a gradual gain in target information. In the present study, we confirmed that the NNC models achieved higher prediction accuracy with a lower number of input biomarkers than traditional modeling methods, including MLR and ANN (Li et al., 2015; Hou et al., 2016; Qu et al., 2017). 
In conclusion, we constructed and validated a NNC-based biomarker panel comprising three miRNAs

(miR-1246, miR-6756-5p, and miR-8073) for early detection of BC. The models were generated using data

from a miRNA microarray database comprising nearly 4000 Japanese female participants. Compared with

the single miRNA biomarker (miR-8073) or the MLR-based miRNA biomarker panel, the NNC-based miRNA biomarker panel was validated to successfully identify BC with significantly higher accuracy of

97.1\%. Given that the dataset used for constructing the biomarker panel was derived from a Japanese

population, further studies using a large cohort of female participants are required to confirm the generalizability of the developed panel to other Asian populations, such as the Chinese population.

\section{REFERENCES}

Bahrami A, Aledavood A, Anvari K, Hassanian SM, Maftouh M, Yaghobzade A, Salarzaee O,

ShahidSales S, Avan A. 2018. The prognostic and therapeutic application of microRNAs in breast cancer:

Tissue and circulating microRNAs. Journal of Cellular Physiology 233(2):774-786 DOI $10.1002 / \mathrm{jcp} .25813$.

Bartel DP. 2009. MicroRNAs: target recognition and regulatory functions. Cell 136(2):215-233 DOI 10.1016/j.cell.2009.01.002.

Bertos NR, Park M. 2011. Breast cancer - one term, many entities? Journal of Clinical Investigation 
222 miR-27b-3p inhibits proliferation and potentially reverses multi-chemoresistance by targeting

223 CBLB/GRB2 in breast cancer cells. Cell Death \& Disease 9(2):188 DOI 10.1038/s41419-017-0211-4.

224 Ding H, Meng J, Zhang W, Li Z, Li W, Zhang M, Fan Y, Wang Q, Zhang Y, Jiang L, Zhu W. 2017.

225 Medical examination powers miR-194-5p as a biomarker for postmenopausal osteoporosis. Scientific

226 Reports 7(1):16726 DOI 10.1038/s41598-017-17075-w.

227 Freiman JA, Chalmers TC, Smith H Jr, Kuebler RR. 1978. The importance of beta, the type II error

228 and sample size in the design and interpretation of the randomized control trial. Survey of 71 "negative"

229 trials. The New England Journal of Medicine 299(13):690-694 DOI 10.1056/NEJM197809282991304.

230 Hamam R, Hamam D, Alsaleh KA, Kassem M, Zaher W, Alfayez M, Aldahmash A, Alajez NM. 2017.

231 Circulating microRNAs in breast cancer: novel diagnostic and prognostic biomarkers. Cell Death \&

Disease 8(9):e3045 DOI 10.1038/cddis.2017.440.

Hannafon BN, Trigoso YD, Calloway CL, Zhao YD, Lum DH, Welm AL, Zhao ZJ, Blick KE, Dooley

WC, Ding WQ. 2016. Plasma exosome microRNAs are indicative of breast cancer. Breast Cancer

Research 18(1):90 DOI 10.1186/s13058-016-0753-x.

Hou S, Wang J, Li Z, Wang Y, Wang Y, Yang S, Xu J, Zhu W. 2016. Five-descriptor model to predict

the chromatographic sequence of natural compounds. Journal of Separation Science 39(5):864-872 DOI

$10.1002 /$ jssc. 201501016.

Jiang X, Tang H, Chen T. 2018. Epidemiology of gynecologic cancers in China. Journal of Gynecologic

Oncology 29(1):e7 DOI 10.3802/jgo.2018.29.e7.

Kozomara A, Griffiths-Jones S. 2014. miRBase: annotating high confidence microRNAs using deep 
243 Li XJ, Ren ZJ, Tang JH, Yu Q. 2017. Exosomal MicroRNA MiR-1246 Promotes Cell Proliferation,

244 Invasion and Drug Resistance by Targeting CCNG2 in Breast Cancer. Cellular Physiology and 245 Biochemistry 44(5):1741-1748 DOI 10.1159/000485780.

246 Li Z, Li Y, Sun L, Tang Y, Liu L, Zhu W. 2015. Artificial neural network cascade identifies multi-P450

247 inhibitors in natural compounds. PeerJ 3:e1524 DOI 10.7717/peerj.1524.

248 Machida T, Tomofuji T, Maruyama T, Yoneda T, Ekuni D, Azuma T, Miyai H, Mizuno H, Kato H,

249 Tsutsumi K, Uchida D, Takaki A, Okada H, Morita M. 2016. miR-1246 and miR-4644 in salivary

exosome as potential biomarkers for pancreatobiliary tract cancer. Oncology Reports 36(4):2375-2381 DOI

251

10.3892/or.2016.5021.

252

Majd SM, Salimi A, Ghasemi F. 2018. An ultrasensitive detection of miRNA-155 in breast cancer via

direct hybridization assay using two-dimensional molybdenum disulfide field-effect transistor biosensor.

Biosensors and Bioelectronics 105:6-13 DOI 10.1016/j.bios.2018.01.009.

255

Myers ER, Moorman P, Gierisch JM, Havrilesky LJ, Grimm LJ, Ghate S, Davidson B, Mongtomery

256

RC, Crowley MJ, McCrory DC, Kendrick A, Sanders GD. 2015. Benefits and Harms of Breast Cancer

Screening: A Systematic Review. JAMA 314(15):1615-1634. DOI 10.1001/jama.2015.13183.

258

Nassar FJ, Nasr R, Talhouk R. 2017. MicroRNAs as biomarkers for early breast cancer diagnosis,

prognosis and therapy prediction. Pharmacology \& Therapeutics 172:34-49 DOI

10.1016/j.pharmthera.2016.11.012.

Qu Y, He Y, Li Z, Chen X, Liu Q, Zou S, Kong C, Liu Y, Gao C, Zhang G, Zhu W. 2017. Constructing 
264

265

266

267

268

269

270

271

272

273

274

275

276

277

278

279

280

281

282

283

284

Shannon P, Markiel A, Ozier O, Baliga NS, Wang JT, Ramage D, Amin N, Schwikowski B, Ideker

T. 2003. Cytoscape: a software environment for integrated models of biomolecular interaction networks.

Genome Research 13(11):2498-2504 DOI 10.1101/gr.1239303.

Shimomura A, Shiino S, Kawauchi J, Takizawa S, Sakamoto H, Matsuzaki J, Ono M, Takeshita F,

Niida S, Shimizu C, Fujiwara Y, Kinoshita T, Tamura K, Ochiya T. 2016. Novel combination of serum microRNA for detecting breast cancer in the early stage. Cancer Science 107(3):326-334 DOI 10.1111/cas.12880.

Siegel RL, Miller KD, Jemal A. 2015. Cancer statistics, 2015. CA: A Cancer Journal for Clinicians 65(1):5-29 DOI 10.3322/caac.21254.

Sun YS, Zhao Z, Yang ZN, Xu F, Lu HJ, Zhu ZY, Shi W, Jiang J, Yao PP, Zhu HP. 2017. Risk Factors and Preventions of Breast Cancer. International Journal of Biological Sciences 13(11):1387-1397 DOI 10.7150/ijbs.21635.

Todeschini P, Salviato E, Paracchini L, Ferracin M, Petrillo M, Zanotti L, Tognon G, Gambino A, Calura E, Caratti G, Martini P, Beltrame L, Maragoni L, Gallo D, Odicino FE, Sartori E, Scambia G, Negrini M, Ravaggi A, D'Incalci M, Marchini S, Bignotti E, Romualdi C. 2017. Circulating miRNA landscape identifies miR-1246 as promising diagnostic biomarker in high-grade serous ovarian carcinoma: A validation across two independent cohorts. Cancer Letters 388:320-327 DOI 10.1016/j.canlet.2016.12.017.

Torre LA, Bray F, Siegel RL, Ferlay J, Lortet-Tieulent J, Jemal A. 2012. Global cancer statistics, 2012. CA: A Cancer Journal for Clinicians 65(2):87-108 DOI 10.3322/caac.21262.

van den Ende C, Oordt-Speets AM, Vroling H, van Agt HME. 2017. Benefits and harms of breast cancer 
285 screening with mammography in women aged 40-49 years: A systematic review. International Journal of

286 Cancer 141(7):1295-1306 DOI 10.1002/ijc.30794.

287 Xiao M, Lou C, Xiao H, Yang Y, Cai X, Li C, Jia S, Huang Y. 2018. MiR-128 regulation of glucose metabolism and cell proliferation in triple-negative breast cancer. British Journal of Surgery 105(1):75-85 DOI 10.1002/bjs.10646.

Yeo SK, Guan JL. 2017. Breast Cancer: Multiple Subtypes within a Tumor? Trends in Cancer 3(11):753-

Zhu W, Kan X. 2014. Neural network cascade optimizes microRNA biomarker selection for nasopharyngeal cancer prognosis. PLoS One 9(10):e110537 DOI 10.1371/journal.pone.0110537. 


\section{FIGURE LEGENDS}

296 Figure 1. AUC distribution and collinearity of miRNA expression. A. Frequency distribution of AUCs.

297 B. Collinearity network of the 82 miRNAs with AUC $\geq 0.95$. An edge represents collinear expression

298

299

300

301

302

303

304

305

between the two miRNAs $\left(\rho^{2}>0.5, p<0.0001\right)$.

Figure 2. Establishment of the NNC model. A. Illustration of the NNC model. L1-L3: Layers 1- 3 of the NNC model; R1246: miR-1246; R6756-5p: miR-6756-5p; R8073: miR-8073; AUC values are shown above the layers. B. ROC curve diagrams of the NNC and MLR models (training set).

Figure 3. Model validation. A. ROC curve diagram of the tenfold cross-validation of the NNC model. B. ROC curve diagram of the NNC and MLR models (validation set). 10FCV: Tenfold cross-validation. C. Accuracy evaluation of miR-8073, MLR, and NNC BC detection (validation set). R8073: miR-8073. ACC: accuracy rate; Se: sensitivity; Sp: specificity. 


\section{Figure 1}

AUC distribution and collinearity of miRNA expression

A. Frequency distribution of AUCs. B. Collinearity network of the 82 miRNAs with AUC $\geq 0.95$.

An edge represents collinear expression between the two miRNAs $\left(\rho^{2}>0.5\right)$. 


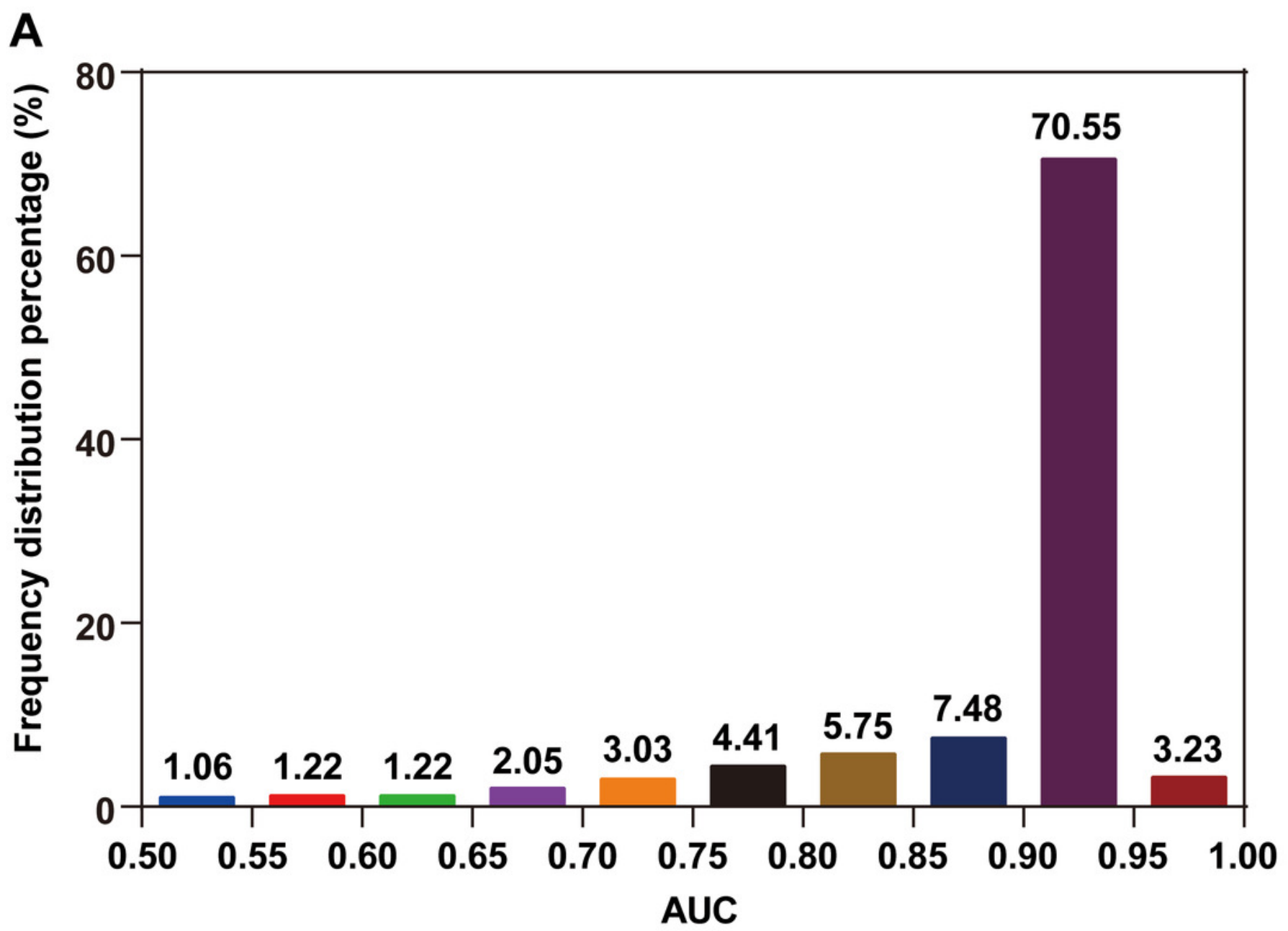

B

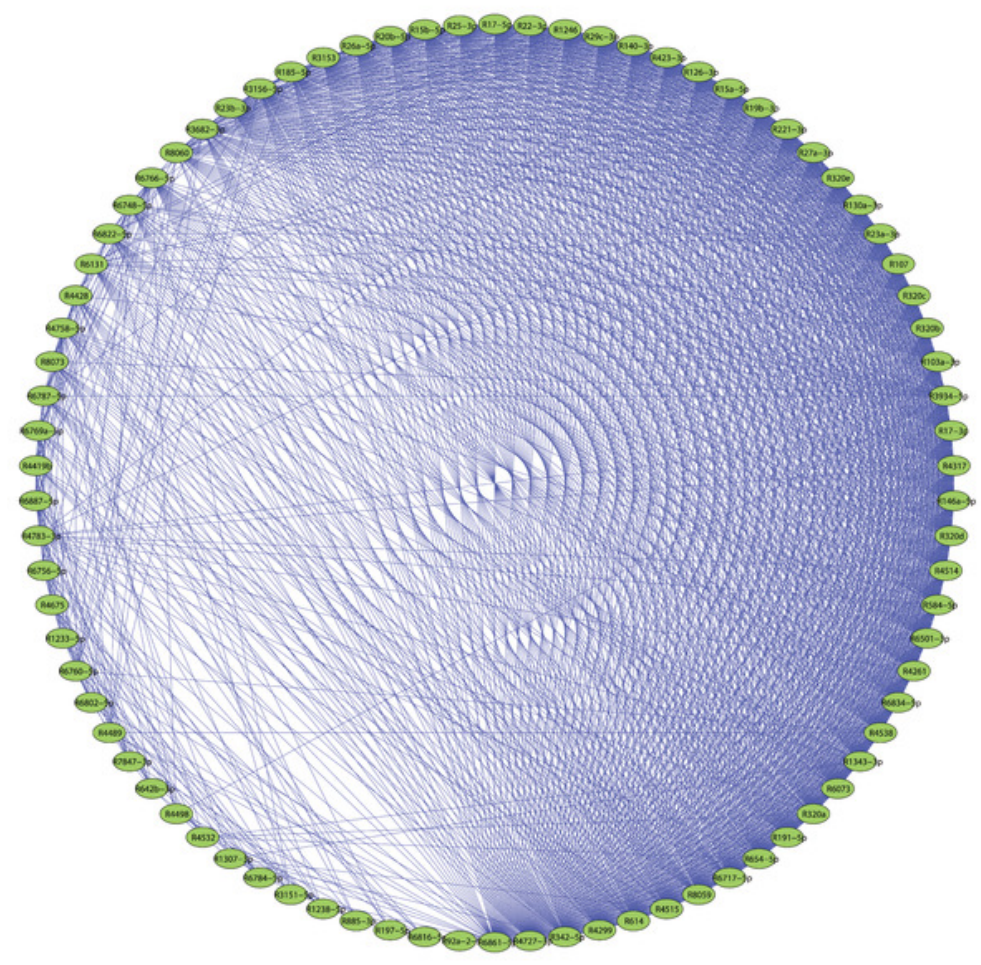


Figure 2

Establishment of the NNC model

A. Illustration of the NNC model. L1-L3: Layers 1- 3 of the NNC model; R1246: miR-1246;

R6756-5p: miR-6756-5p; R8073: miR-8073; AUC values are shown above the layers. B. ROC curve diagrams of the NNC and MLR models (training set).

A

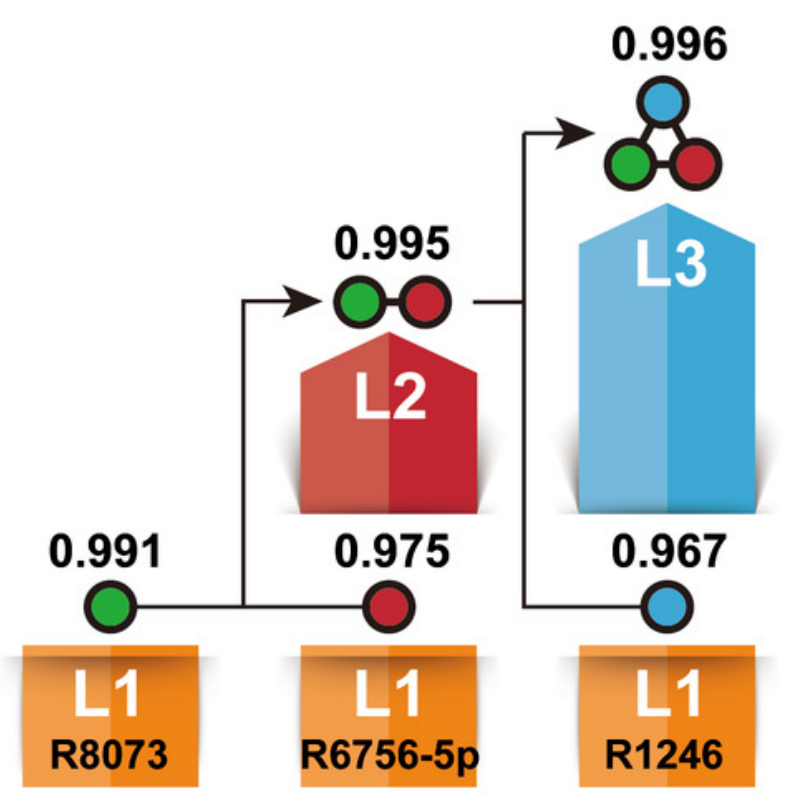

B

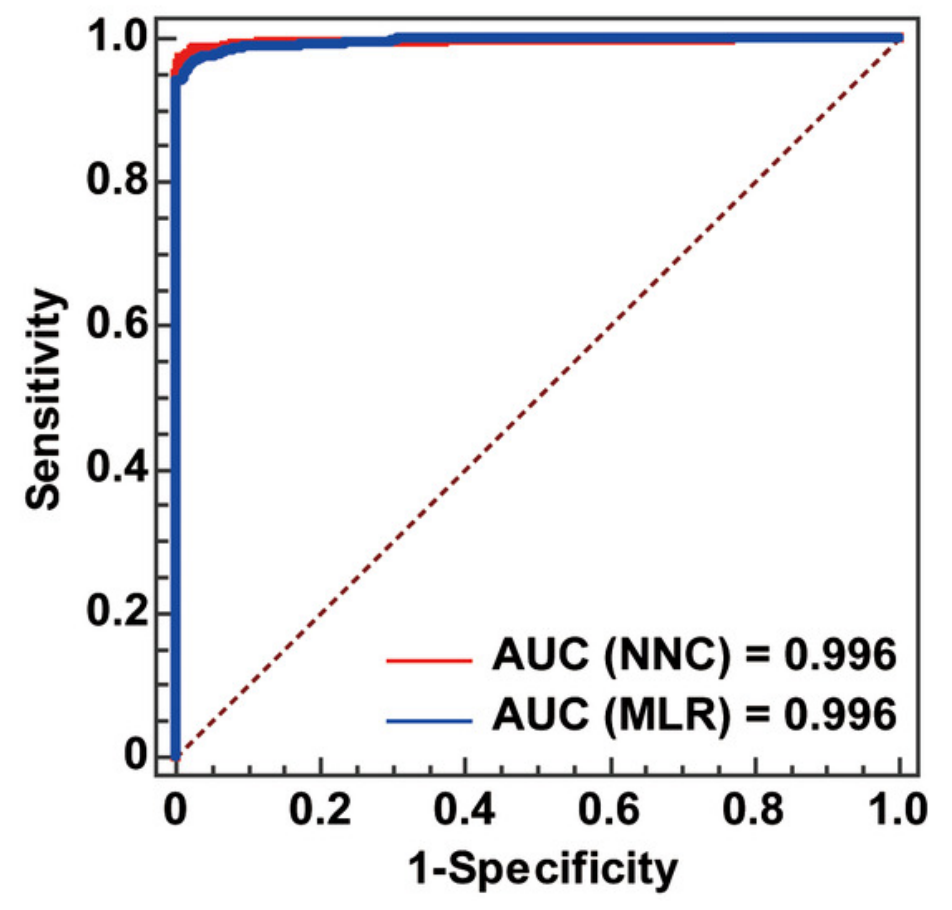


Figure 3

Model validation

A. ROC curve diagram of the tenfold cross-validation of the NNC model. B. ROC curve diagram of the NNC and MLR models (validation set). 10FCV: Tenfold cross-validation. C. Accuracy evaluation of miR-8073, MLR, and NNC BC detection (validation set). R8073: miR8073. ACC: accuracy rate; Se: sensitivity; Sp: specificity. 
A

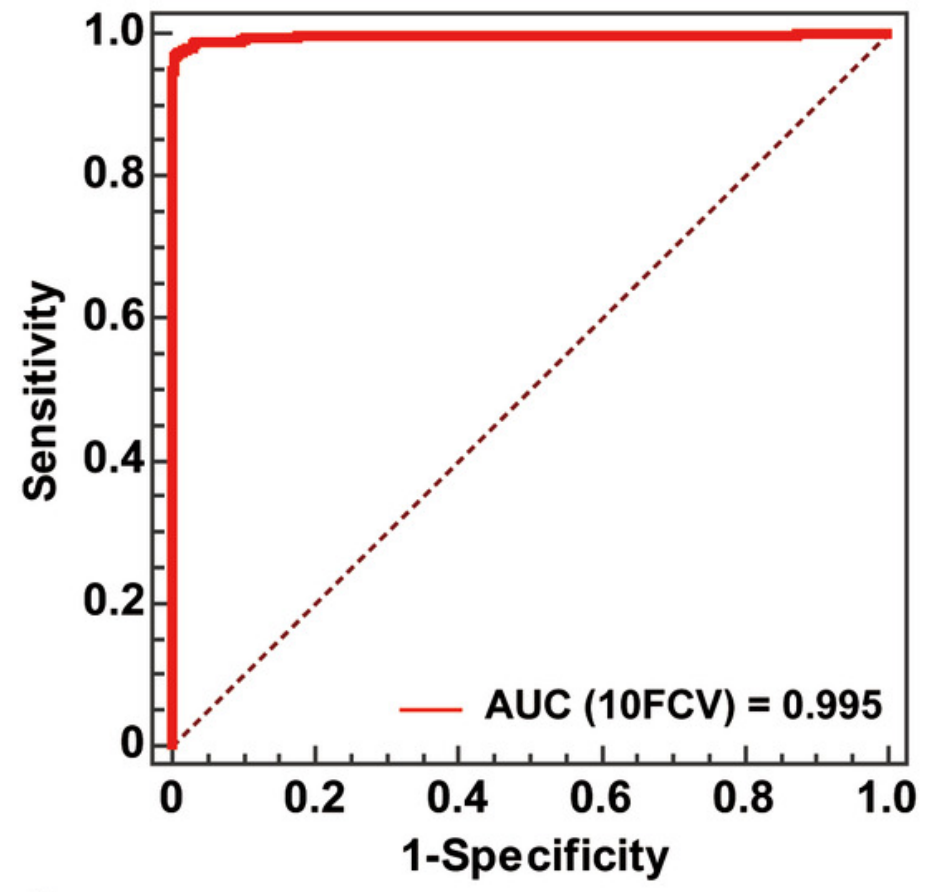

C

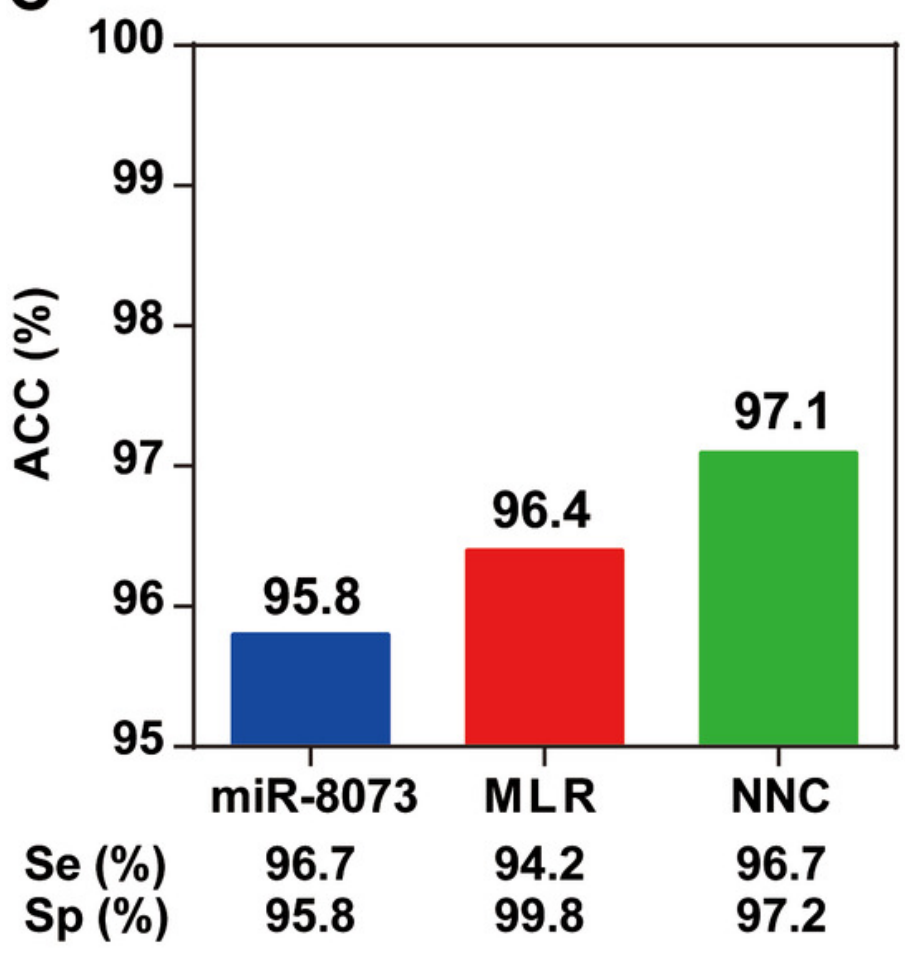

B

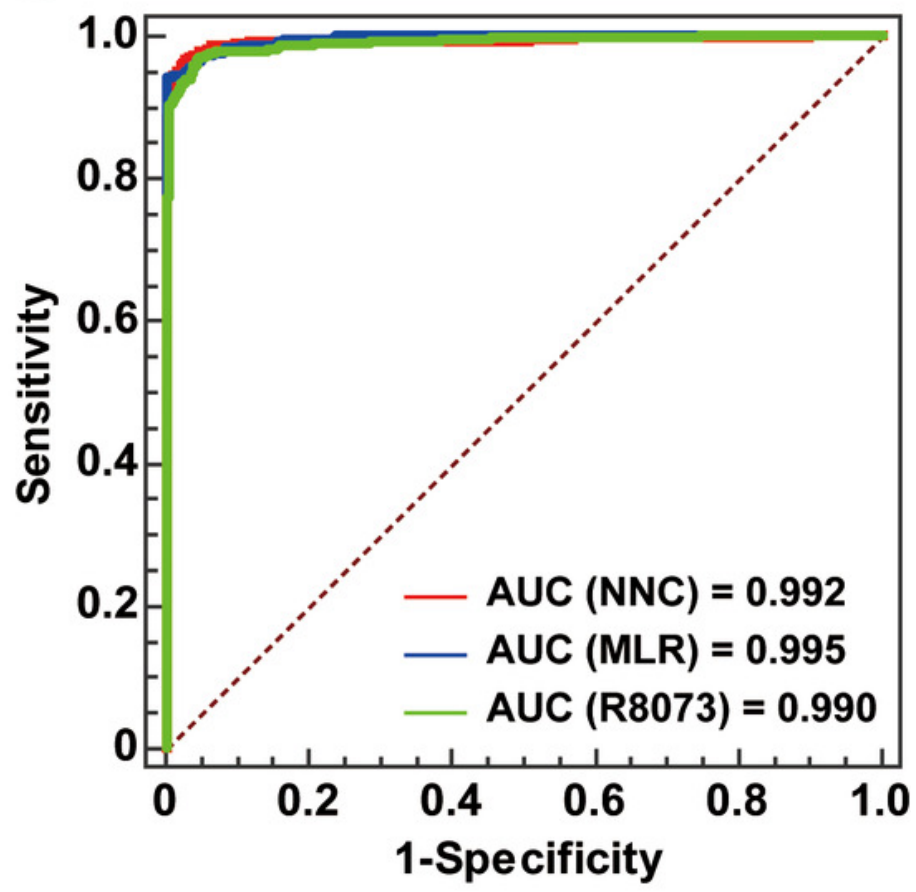




\section{Table $\mathbf{1}$ (on next page)}

ROC curve analysis of individual miRNAs (training set) 
1 Table 1. ROC curve analysis of individual miRNAs (training set).

\begin{tabular}{|l|l|l|l|l|}
\hline miRNA ID & AUC & Sensitivity (\%) & Specificity (\%) & ACC (\%) \\
\hline miR-197-5p & 0.961 & 90.7 & 95.9 & 94.2 \\
\hline miR-1238-5p & 0.964 & 90.3 & 97.1 & 94.9 \\
\hline miR-1246 & 0.967 & 89.8 & 91.7 & 91.1 \\
\hline miR-3156-5p & 0.976 & 89.8 & 96.1 & 94.0 \\
\hline miR-4532 & 0.968 & 89.8 & 98.7 & 95.8 \\
\hline miR-6748-5p & 0.962 & 90.2 & 90.3 & 90.3 \\
\hline miR-6756-5p & 0.975 & 92.7 & 97.2 & 95.7 \\
\hline miR-8073 & 0.991 & 95.7 & 97.6 & 97.0 \\
\hline
\end{tabular}

2 
Table 2 (on next page)

Comparison between NNC and MLR models (training set) 
1 Table 2. Comparison between NNC and MLR models (training set).

\begin{tabular}{|l|l|l|l|l|}
\hline Model & AUC & Sensitivity (\%) & Specificity (\%) & ACC (\%) \\
\hline Layer 1 of NNC & 0.991 & 95.7 & 97.6 & 96.9 \\
\hline Layer 2 of NNC & 0.995 & 95.8 & 98.5 & 97.6 \\
\hline Layer 3 of NNC & 0.996 & 97.3 & 99.1 & 98.5 \\
\hline MLR & 0.996 & 96.5 & 97.9 & 97.4 \\
\hline
\end{tabular}

2 